\title{
ERMO $^{2}$ algorithm: an energy efficient mobility management in mobile cloud computing system for 5G heterogeneous networks
}

\author{
L. Pallavi' ${ }^{1}$ A. Jagan ${ }^{2}$, B. Thirumala Rao $^{3}$ \\ ${ }^{1,3}$ Department of Computer Science and Engineering, KL University, India \\ ${ }^{2}$ Department of Computer Science and Engineering, BVRIT, India
}

\begin{tabular}{l}
\hline \hline Article Info \\
\hline Article history: \\
Received Jun 10, 2018 \\
Revised Nov 10, 2018 \\
Accepted Dec 5, 2018 \\
\hline
\end{tabular}

Keywords:

Best Cloud

Energy Efficient

Heterogeneous Network

Mobile Cloud Computing

Mobility Management

\begin{abstract}
Recently, mobile devices are becoming the primary platforms for every user who always roam around and access the cloud computing applications. Mobile cloud computing (MCC) combines the both mobile and cloud computing, which provides optimal services to the mobile users. In nextgeneration mobile environments, mainly due to the huge number of mobile users in conjunction with the small cell size and their portable information's, the influence of mobility on the network performance is strengthened. In this paper, we propose an energy efficient mobility management in mobile cloud computing (E2M2MC2) system for 5G heterogeneous networks. The proposed E2M2MC2 system use elective repeat multi-objective optimization (ERMO2) algorithm to determine the best clouds based on the selection metrics are delay, jitter, bit error rate (BER), packet loss, communication cost, response time, and network load. ERMO2 algorithm provides energy efficient management of user mobility as well as network resources. The simulation results shows that the proposed E2M2MC2 system helps in minimizing delay, packet loss rate and energy consumption in a heterogeneous network.
\end{abstract}

Copyright (ㅇ 2019 Institute of Advanced Engineering and Science. All rights reserved.

\section{Corresponding Author:}

L. Pallavi,

Department of Computer Science and Engineering,

KL University,

Vaddeswaram, Guntur, 522502, A.P, India.

Email: pallavi503@gmail.com

\section{INTRODUCTION}

Cloud computing is generally employs for the computing resources which are distributed by the help of network. Suppose, if the functions and data are maintained on the internet then it offering on-demand access [1]. Applications are normally execute on a remote server. Afterward, it is transmitted to the consumer. Mobile-learning is mostly take place if the populaces are far from their offices or classrooms. After the school or office, several populaces are chosen listen music, radio news, or sports programs. Moreover, they desire to learn mobile devices, DVD/CD Players, videotapes, computers installed by means of learning software or computers with high speed access to the Internet after they get home which are all based on e-learning [2]. Generally, the mobile phones are employs for communications with further populace, not for learning intention. M-learning is normally an innovative technology for learning but it does not swap conventional learning [3]. Here, the mobile phones are containing a diminutive memory and storage capability, which are restricts the length of emails, the opening of attachments, and the employ of tabs or numerous windows [4].

Recently, the access of cloud servers through mobile phone is considered as a general and trendy. In 2018, the cloud applications are encountered $90 \%$ of mobile data traffic. Generally, the mobile devices are containing numerous obtainable storage services like Dropbox, iCloud, Google Drive, and Sky drive [5]. 
The fundamental technology of cloud computing is used to centralize the computing, services, and precise applications by the help of service which is distributed like water, gas or electricity to consumer. Therefore, the mixture of ubiquities mobile network and cloud computing is used to engender an innovative computing approach, which is known as MCC [6]. In modern computer regions, the mobility is turn out to be a trendy metrics. Normally, the mobile devices like Smartphone, PDA, GPS Navigation and laptops are expanded by various mobile computing, networking and security technologies [7]. Here, expansion rate of compound program is quicker than the expansion rate of computing resources of mobile devices which are based on the expansion of computing-intensive applications [8]. Additionally, the surfing of Internet is turned out to be easier by the expansion of wireless technology like WiMax, Ad Hoc Network, and WIFI, but it not restricted by means of cables. Therefore, those mobile devices are established as their foremost option of functioning and entertainment in people lives [9]. Based on the analysis of Juniper, the cloud computing related mobile software and application are anticipated to increase 88\% per annum from 2009 to 2014. This expansion is also generating US 9.5 billion dollars in 2014 [10], [11].

The foremost benefit of MCC is to conquer difficulties of mobile computing which are associated to the presentation, atmosphere, and protection [12]. Mobile computing is generally derived from three foremost perceptions like hardware, software and communication. The perception of hardware is indicating mobile devices as smart phone and laptop, or their mobile components. The difficulty of communication is mainly encompassing the communications of mobile networks, protocols and data deliverance. Some of the attributes of mobile computing are specified as follows [13]: In mobile computing network, the mobile nodes are used to make association with others. Moreover, the predetermined nodes in wired network are take place by mobile support station (MSS) for the period of moving.

Normally, the employing of mobile nodes in network is not exclusive. Therefore, this network can be a wired network through high-bandwidth, low-bandwidth, or even in position of disconnected [14]. According to restriction of battery power, charge of wireless communication, network conditions and etc, the mobile nodes are not constantly maintaining the association, but detach and reliable through the wireless network is take place as inactively or actively [15]. Here, the servers, access points, and further MSS are facilitating a sturdy send/receive capability. This capability in mobile nodes is somewhat weak. Therefore, the communication bandwidth and overhead among downlink and uplink are inconsistency [16]. A mobile computing network system is generally recognized from terminals, networks, database platforms, and applications expansion for defense by reason of signal is vulnerable to obstruction and snooping.

In contrast to the conventional wired network, the mobile computing network is encompassing diverse difficulties and challenges in dissimilar features like signal interruption, protection, hand-off postponement, restricted power and low computing capability [17]. Three foremost possessions are considered for the limited attributes of mobile networks: there is a requirement for combining possessions from numerous devices because mobile device is encompassing restricted resource than conventional wired networks [18]. Physical uniqueness of network is necessary to incorporated like stable routes, and etc [19]. Energy limitations are significant in mobile network because the recent applications are necessitating additional battery [20]. On the other hand, the attributes of mobility is also containing the disadvantage of rapidly energy reduction and unbalanced method [15]-[20]. For this reason, the proposed energy efficient mobility management in mobile cloud computing (E2M2MC2) system is required for next generation 5G heterogeneous networks. The main of proposed E2M2MC2 system is to compute the optimal best cloud among others, which provides connection less service to end mobile users; and the user mobility is managed by the back track searching (BTS) algorithm with congestion control also.

The remainder of this paper is organized as follows. Section 2 discusses recent related work. In Section 3, we present the problem methodology and system model of proposed work. Section 4 describes the working function of proposed E2M2MC2 system in detail with proper mathematical models. Section 5 provides the simulation results and performance analysis with proper test scenarios. The paper concludes in Section 6.

\section{RELATED WORKS}

Sardis et al. [21] have offered a possible circumstance for to generate travel congestion difficulties on the Internet by reason of elevated bandwidth media services and consumer mobility. Here, the logical structure is employs to examine the feature which concern the Quality of Experience (QoE) and QoS for VoD services in a mobile atmosphere. The cloud-related services are generally providing reasonable and centralized computing resources and mobile devices. Moreover, it is also requiring centralized resources for to generate their need of processing power. Afterward, the examination of service deliverance structure is used to conquer a difficulty through the employ of service populating procedure and Cloud services. 
Qi et al. [22] have anticipated a service-responsive position method which is employs to identify the occurrence and position of mobile device devoid of conventional cyclic registration update. Internet protocol (IP) in multimedia subsystem (IMS) is mainly used to acquire the position and circumstances information of mobile devices by the help of registration progression. Here, a binding was formed by means of serving-call session control function (S-CSCF) which is derived from preliminary registration among public consumer identity and IP address of mobile device. The S-CSCF and proxy call session control function (P-CSCF) is generally used to maintain the consumer registration position like the timer which is specifying the 'expires' limitation.

Gani et al. [23] have anticipated a thematic taxonomy which is used for the classification of existing interworking and mobility procedure of wireless data networks. Here, the proposition and significant features of existing faultless connectivity procedures are examined as effectively. In heterogeneous wireless data networks, the considered two significant units of faultless connectivity are interworking and mobility procedures. Here, the interworking procedure is used to incorporate various wireless data networks for dissimilar OSI layers which are facilitating node mobility in heterogeneous wireless data networks for retaining connectivity. In the progression of mobility, numerous procedures are employing proactive mobility to calculate the possible relinquish for to evade package failure and association failure. Moreover, the further procedures are offering reactive mobility for lesser signaling transparency handover. The foremost intention of method is to diminish the latency, package failure, and multimodal. Here, QoS is maintaining signaling transparency and mobility for the optimization difficulty.

Chen et al. [24] have anticipated mobility-driven network (MDN) which is derived from the examination of development phases. Here, the mobility organization is classified as three segments such as one attribute in a precise network, mobility in a common competence of networks and mobility. Suppose, if the mobility is supporting the environment then obtainable mobility technology is illustrating the problem of task redundancy, system complication, inadequacy, and scalability. MDN is mainly employs the design of viewpoint, task orientation representation, and protocol orientation representation for a high-level concept of key task, network entities, and scientific values.

Hu et al. [25] have mainly focusing the mobility problem in the cloud data center which is used to convene diverse necessities of Internet services to supply enhanced consumer experience. Generally, the virtual machines are competent to transfer from one position to another. The foremost difficulty of IP mobility was a relocation of virtual machine (VM) in IP subnets. It is demonstrating the mobility-based cloud data center network at structural design which is derived from the design of MDN. Here, the Entity-identity/Location-identifier is decoupling to establish a position executive to plan the Entity-identity at the existing position of VM. Moreover, the mapping table is used to rearrange the VM for faultless service which is offered by means of cloud data center.

Junior et al. [26] have offered the mobile offloading system (MOSys) which is generally employs to retain and maintain the function of mobile nodes. The basic system structural design is mainly used to support the software defined network (SDN) for the mobility organization task. The operational situation of cache related organization system is reliant on the off-load reaction time. The progression of middleware is employs to preserve the data off-load, profiling services, cloud detection, and application consumption. The system was estimated by means of mobility impact limitation on the off-load presentation at the implementation arrangement in the subsequent production mobile devices. The MOSys mobility organization system is mainly used to control the elevated density of mobile nodes through the collection of low expenditure and energy competent conversion from one position to another position.

Kim et al. [27] have offered a traffic organization process by means of mobile edge cloud. The mobile edge cloud is generally situated in the mobile edge network for to observe the position of mobile terminals. Therefore, it turns out to be probable for control the traffic of mobile terminals efficiently through the network. This process is normally controls the video traffic from content server in the Internet which is based on the edge network position and mobility of a mobile terminal. Moreover, it offers the video traffic to the mobile terminals. This process is employing the mobile edge cloud for to develop the QoE of mobile video consumer.

Seo et al. [28] have anticipated the cloud communications for ubiquitous computing environment mobile application (CI-UCEMA), which encompasses three layers like cloud service layer (CSL), M2M service layer (MSL) and ubiquitous service layer (USL). The M2M is including IoT services layer (MSL) for to diminish the complication of both the enhancement and maintenance of IoT systems. It is mainly demonstrated by means of enhanced interoperability and the employ of standard process. Protection is also accomplished by means of device management and provisioning. The MSL was dependable for preventing the consumer from fundamental complication and inconsistency in self-modification atmosphere by the help of mobility and adaptation at CDPS and CIMS. 


\section{PROBLEM METHODOLOGY AND SYSTEM MODEL}

\subsection{Problem methodology}

Aissioui et al. [29] have anticipated an elastic method which is derived from SDN/Open-Flow structural design and a control plane structure. They are generally modified for mobile cloud computing systems and Follow Me Cloud (FMC)-related systems. The circumstance of 5G mobile networks are mainly employs to manage the plane structure for MCC-related systems where mobile nodes and network services are indicating limitation of activities and relocations. Moreover, the dissimilarity of centralized method through solitary SDN regulator is facilitating to allocate the SDN/Open-Flow control plane on a two-level hierarchical structural design which are containing first stage among a global regulator of G-FMCC and second stage among numerous local regulator. The assessment consequences are acquired by means of investigation. Additionally, this explanation is making sure the enhanced control plane organization, presentation maintenance, and network resource conservation. MCC is a convergent technology comprised of three cornerstone heterogeneous technologies, namely mobile computing, cloud computing, and networking. The upcoming heterogeneous $5 \mathrm{G}$ network emphasizes on a dramatic increase in the transmission rate of MCC traffic. With more users operating at high rates, the type of data shared over the network is going to be complex and a majority of it will include video traffic. Such complex structure of traffic and heavy load over the components of the network are difficult to control. Further, the mobility of users adds up to this issue and makes it difficult to manage and operate the network without any breakdown. Thus, it is important to control traffic as well as manage the mobility of users to provide efficient communication, which can support video traffic at high delivery rates. Additionally, the basic parameters affect the performance of network as the vast variety of mobile devices with different operating systems (OSs), platforms, and wireless network standards. The upcoming 5G networks aim at providing high speed communications to users irrespective of their movement. With an increase in the number of devices and the network attaining its peak size, due to dense deployment, it becomes important to manage and control mobility for efficient communication. Mobility management requires multiple operations at the same instance, which include optimal route selection, mobile anchor support, user pattern identification, and service handoffs.

An energy efficient mobility management in mobile cloud computing (E2M2MC2) system utilizes the optimal cloud selection to obtain energy efficient mobility management. The main contributions of proposed E2M2MC2 system are summarized as follows:

a. In E2M2MC2 system, an elective repeat multi-objective optimization (ERMO ${ }^{2}$ ) algorithm used to compute the best cloud among others in the network.

b. Various parameters used for best cloud election process are: delay, jitter, BER, packet loss, communication cost, response time, and network load.

c. Finally, the performance of proposed E2M2MC2 system is compared with the existing simulation results shows that the proposed E2M2MC2 system helps in minimizing handover delay, packet loss, average queuing delay, and device lifetime in a heterogeneous network.

\subsection{System model of proposed E2M2MC2 system}

The system model of proposed E2M2MC2 system is shows in Figure 1, which uses the follow me cloud (FMC) concept, which allows the relocation of services provided to users depending on their movements. Services are therefore always provided from data center locations that are optimal for the current locations of the users. This provides users with improved QoS/QoE, at the same time and it allows preserving operators' network resources by escaping network traffic to data centers through the nearest points compared with users' locations. Another advantage of FMC technology is that migration of services is seamless and transparent to users. MCC framework use both the information stockpiling and the information handling happen outside of the cell phone. Concerning definition, mobile applications move the processing force and capacity from the cell telephones to the Cloud. It might be thought the consolidation of the cloud computing and mobile environment. 


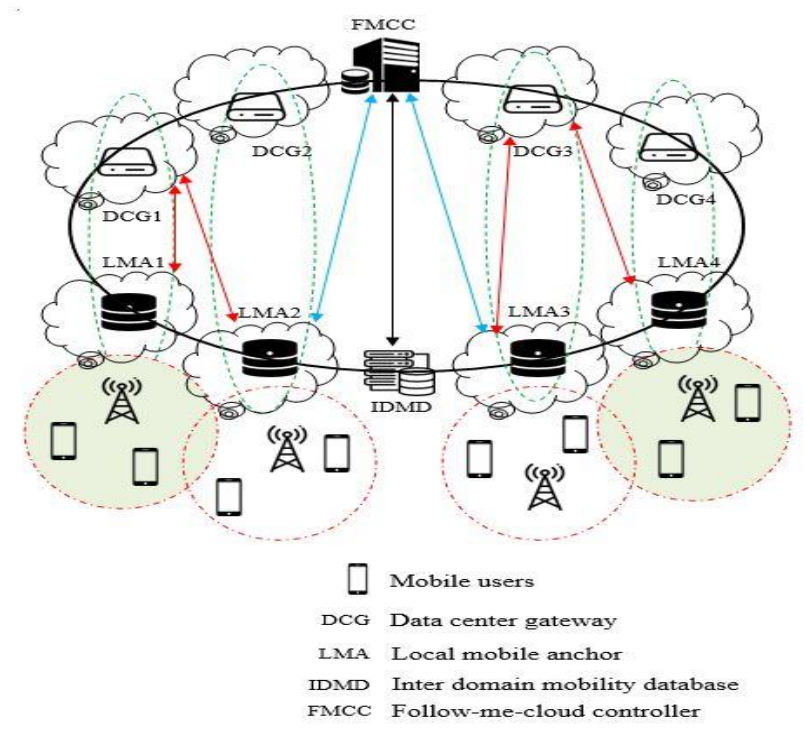

Figure 1. System model of proposed E2M2MC2 system

\section{ENERGY EFFICIENT MOBILITY MANAGEMENT IN MOBILE CLOUD COMPUTING (E2M2MC2) SYSTEM}

The movement is tenacious and changed change influenced by the creature's own particular locomotors attempts passing on them to new typical condition. Creature movement is the fairly long-confine change of people, by and large talking on a typical begins. It is an inevitable ponder that can be found in all enormous creature parties, for example, birds, mammals, fish, reptiles, amphibians, insects, and crustaceans. The multi-objective optimization (MOO) algorithm [30] can be divided into Diaspora process and updating process. The animal Diaspora process computes the groups of animal move from one position to next and the animal update process computes position changed animals are updated by the probabilistic method. MOO algorithm with the Diaspora process and population updating process are used to compute a satisfactory solution. The proposed elective repeat multi-objective optimization $\left(\mathrm{ERMO}^{2}\right)$ algorithm inspired from conventional MOO algorithm [30] with a new migration process by establishing a living area by the best fitness value owned animal and animals migrate from current locations into this new living area to simulate animal Diaspora process. We consider $\mathrm{N}$ animals that live in living area, some individuals move randomly and their position updated, and then we calculate the best position of animals by fitness function and record it. But the amount of food or water gradually diminished as the time wore on, and some animals migrate from the current areas which have no food and water to a new area with abundant food and water.

ERMO $^{2}$ algorithm begins with an initialization process, for this work, we consider the animal as sensor nodes and their position as network position. Let set of $\mathrm{N}$ sensor nodes and their positions are $P_{1}, P_{2}, P_{3}, \ldots, P_{N}$; each animal position $P_{i}$ is a $1 \times(n \times d)$-dimensional vector, where $n_{\text {the number of }}$ clusters and $d$ is the dimension of the test set. The clusters $P_{i}^{*}=\left(p_{i_{1}}, p_{i_{2}} \ldots p_{i_{d}}\right)$, where $i=1,2, \ldots, n$; each cluster is $1 \times \mathrm{D}$-dimensional vector, and the lower bound of the centers is the minimum of each column in test set $t_{n \times d}$,denotes $t_{l}=\min \left(t_{1}, t_{2}, \ldots, t_{d}\right)$, and the upper bound of the centers is $t_{u}=\max \left(t_{1}, t_{2}, \ldots, t_{n}\right)$. Sensor nodes are randomly and uniformly distributed between the pre-specified lower initial parameter bound $l_{\text {and }}$ the upper initial parameter bound $u$. Once the neighborhood topology has been constructed, we select one neighbor randomly and update the position of the individual according to this neighbor as follows:

$$
p_{i, j}=t_{l_{j}}+r_{i, j}[0,1] \cdot\left(t_{u_{j}}-t_{l_{j}}\right)
$$

where $i=1,2, \ldots, n$ and $j=1,2, \ldots, n \times d, r_{i, j}[0,1]$ represents the random numbers between 0 to 1 . During the Diaspora process, because of nodes movement in the network area, some parts of the networks are lacking due to the condition change, and some nodes moving from the current position to new position 
depends on moving action. During the updating process, the algorithm computes how nodes leave the cluster and some join in the new population. Individuals will be replaced by some new nodes with a probability $P_{\text {new }}$ .The probability is used according to the quality of the fitness. We sort fitness in descending order, so the probability of the individual with best fitness is $1 / \mathrm{N}$ and the individual with worst fitness, by contrast, is 1 .

After compute the new solution $p_{i, j+1}$, it will be evaluated and compared with the $p_{i, j}$ and we choose the individual with a better objective fitness and denotes as follows:

$$
P_{i}= \begin{cases}p_{i, j} ; & \text { if } f(i, j) \text { is better than } f(i, j+1) \\ p_{i, j+1} ; & \text { Otherwise }\end{cases}
$$

In this work, we use Rastrigin's function for fitness computation and define as follows:

$$
f(i, j)=\sum_{i=1}^{n}\left[p_{i}^{2}-10 \cos \left(2 \pi \mathrm{p}_{\mathrm{i}}\right)+10\right]
$$

where the range variables denotes as $-5.12 \leq \mathrm{p}_{\mathrm{i}} \leq 5.12$. Rastrigin's function is based on cosine modulation to produce many local minima. The parameters for cloud selection are: delay (D), jitter (J), BER, packet loss $(\mathrm{PL})$, communication cost $(\mathrm{C})$, and network load $(\mathrm{nL})$. The parameters are time varying function and it optimized by the proposed $\mathrm{ERMO}^{2}$ algorithm. The selection criterion for best optimal cloud as follows,

$$
\text { Best cloud }=\min \{D, J, B E R, p L, C, n L\}
$$

Delay is defined as the average of the sum of the distances of all the mobile users from their selected cloud.

$$
D=\sum_{i=1}^{n} \operatorname{dis}\left(m_{n}, \text { Best cloud }\right)
$$

Average sink distance is defined as the ratio of distance between IDMD to the DCG as follows,

$$
A v g_{d}=D \cdot \operatorname{dis}(\text { Best cloud }, D C G)
$$

All mobile users consume some energy due to their processing time and to consume less energy. The average communication distance and DCG is close to all the mobile users. To minimize the average delay and DCG distance of all the Best cloud by optimal selection approach i.e.

$$
T_{D}=\sum_{i=1}^{n}\left(D+A v g_{d}\right)
$$

Simply, jitter is defined as the variation in delay of received packets. Due to network congestion, improper queuing, or configuration errors, this steady stream can become delay between each packet can vary instead of remaining constant. Let us consider two consecutive packets of the tagged traffic, $P_{0}$ and $P_{1}$. For packet $P_{j}, j=0,1$ and node $\mathrm{k}=1, \ldots, \mathrm{n}$, let $\tau$ in $\mathrm{j}(\mathrm{k})$ and $\tau$ out $\mathrm{j}(\mathrm{k})$ be the arrival and departure times of $p_{j}$ at node $\mathrm{k}$, let $w_{j}(k)$ be the waiting time of $p_{j}$ at node $\mathrm{k}$, and finally let $\Delta k=w_{1}(k)-w_{0}(k)$ be the variation of the inter-packet delay at node $\mathrm{k}$. The end-to-end jitter is defined as,

$$
J(n)=E\left(\sum_{i=1}^{n} \Delta_{k}\right)
$$


End-to-end jitter is the expected absolute value of the sum of inter-packet delay variations introduced by each mobile user along the path between the source and the destination. The bit error ratio (BER) is the number of bit errors divided by the total number of transferred bits during a studied time interval. Bit error ratio is a unit less performance measure, often expressed as a percentage. Packet loss (PL) refers to the number of packets lost to reach the destination when being transmitted across the network. It is one of the important QoS parameter for real time application such as cloud gaming as of a case in our analysis. The communication cost (C) is sum of the cost of all the tasks implementing that algorithm. We shall focus on the cost as the way to measure the efficiency of algorithm and the amount of time it takes each task to execute when estimating the running time of an algorithm.

The network load $(\mathrm{nL})$ is the weighted whole of whatever is left of the load of individual users of the baffling number of users. The straggling stays of the load of individual user is depicted as the made holding up criticalness out of the user. By uprightness of the mobile users, the bundle might be retransmitted to ensure the right transport. The criticalness of each inside and their condition in like way used to pick remaining load of the entire user. We consider the straggling bits of the load of the entire mobile user regulate as the aggregate of the weighted extra lifetime of all users in the network. Thus, the nL of the mobile users as follows:

$$
n L=W_{a} \sum_{i=1}^{n} \frac{\max \left(\Delta_{k}+J(T)\right)}{D+A v g_{d}}
$$

where ${ }^{W}$ represents the weight factor of each mobile user and represented by,

$$
W_{a}=c \frac{1}{A v g_{d}}
$$

where $\mathrm{c}$ represents the constant.

The working flow of entire proposed E2M2MC2 system illustrates in Figure 2.

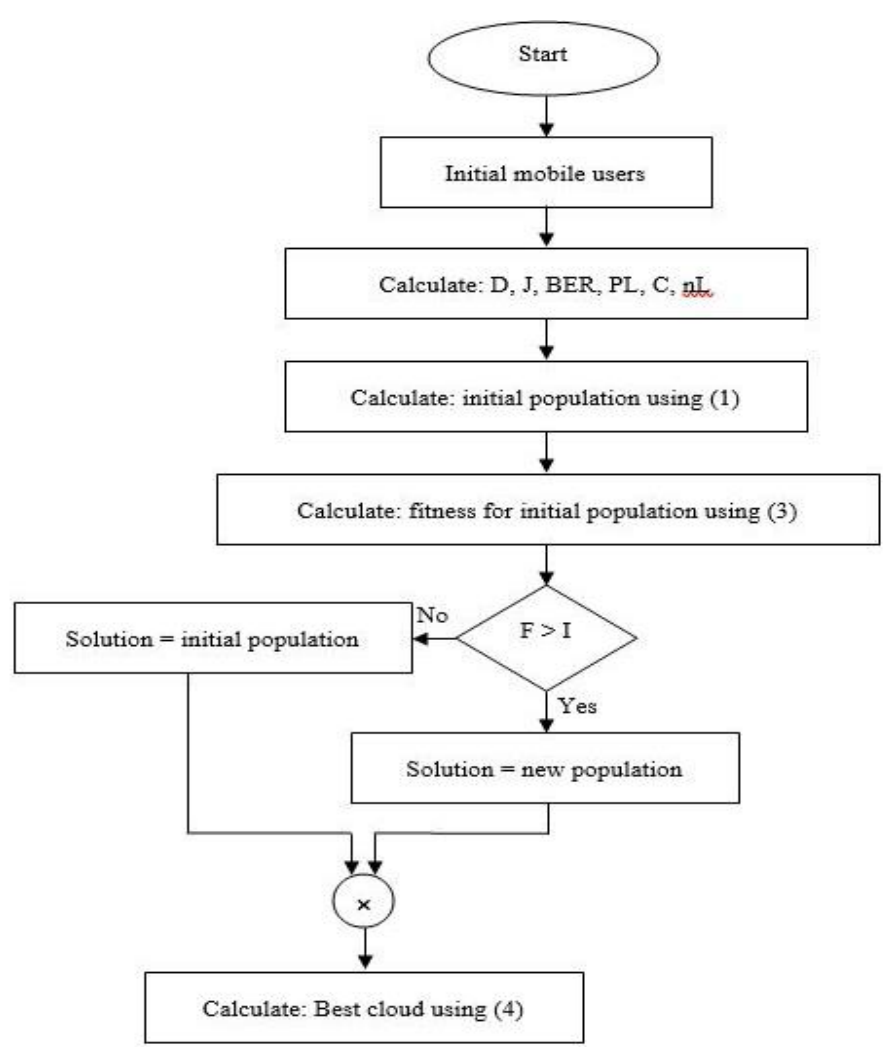

Figure 2. Working flow of proposed E2M2MC2 system cloud selection using ERMO ${ }^{2}$ algorithm 


\section{PERFORMANCE ANALYSIS}

The mobile cloud computing architectures is analyzed for the performance point of view. Mobile cloud architecture increases resource availability by leveraging large number of nearby mobile devices in public places like shopping mall, cinema, and airport service availability is increasing noticeably. It also enhances security because of the dynamic partitioning of the communication channel in the between mobile device and cloud server. In this section, we present the evaluation of our energy efficient mobility management in mobile cloud computing (E2M2MC2) system and it compared with the existing distributed follow me cloud controller (DFMCC). The performance of proposed E2M2MC2 system is analyzed by the different testing scenarios: impact of mobile user density and their speed. The number of mobile users is varied from 30 to 110 in first test and the mobile user speed is varied from 20 to $100 \mathrm{~ms}$ in the second scenario. For this testing, we use four DCG and LMA, one IDMD and one FMCC with high density mobile users. Both tests are implemented in Network Simulator (NS2) tool with $1000 \times 1000 \mathrm{~m}^{2}$ network size. The simulation parameters are summarized in Table 1.

Table 1. Simulation Parameters

\begin{tabular}{cc}
\hline Parameters & Values \\
\hline Number of mobile users & $30,50,70,90,110$ \\
Mobile user speed (ms) & $20,40,60,80,100$ \\
Number of DCG & 4 \\
Number of IMA & 4 \\
Number of IDMD & 1 \\
Number of FMCC & 1 \\
Network size & $1000 \times 1000 \mathrm{~m}^{2}$ \\
Traffic model & Constant bit rate \\
Simulation time (s) & 100 \\
\hline
\end{tabular}

\subsection{Impact of mobile users}

In this scenario, we varying the number of node from 30 to 110 with the fixed speed as $60 \mathrm{~ms}$ and the performance of proposed E2M2MC2 is compared with the existing DFMCC system. Figure 3 shows the packet loss rate of proposed E2M2MC2 and existing DFMCC system. The plot clearly depicts the packet loss rate of proposed E2M2MC2 system is very low in terms of $41 \%$ less compared to existing DFMCC system.

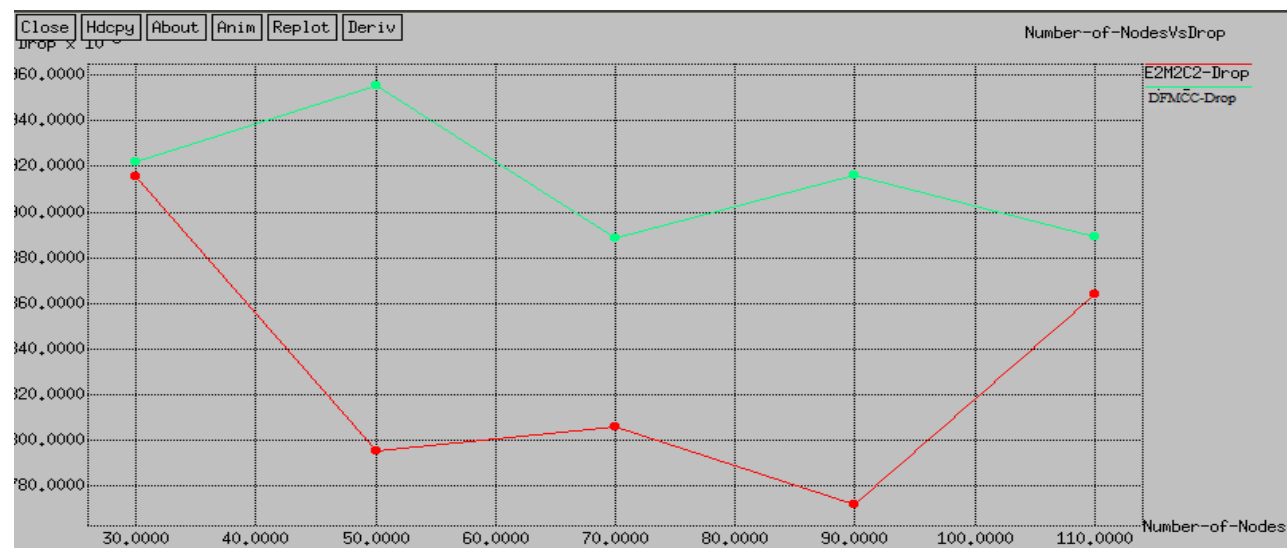

Figure 3. Packet loss rate comparison with impact of mobile users

Figure 4 shows the energy consumption of proposed E2M2MC2 and existing DFMCC system. The plot clearly depicts the energy consumption of proposed E2M2MC2 system is very low in terms of 39\% less compared to existing DFMCC system. 


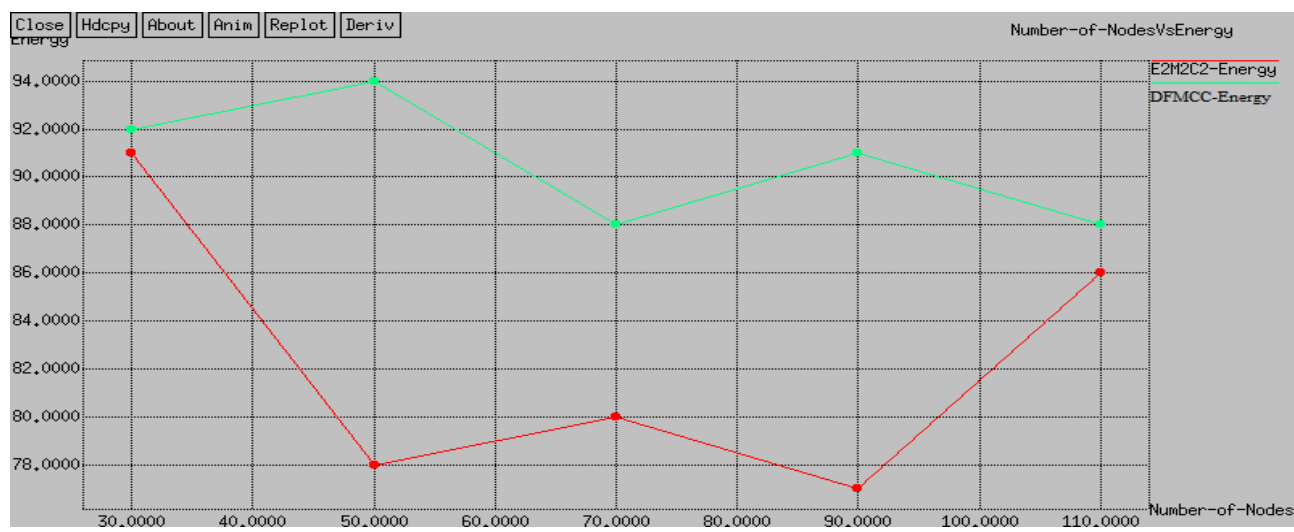

Figure 4. Energy consumption comparisons with impact of mobile users

Figure 5 shows the throughput of proposed E2M2MC2 and existing DFMCC system. The plot clearly depicts the throughput of proposed E2M2MC2 system is very high in terms of $24 \%$ high compared to existing DFMCC system.

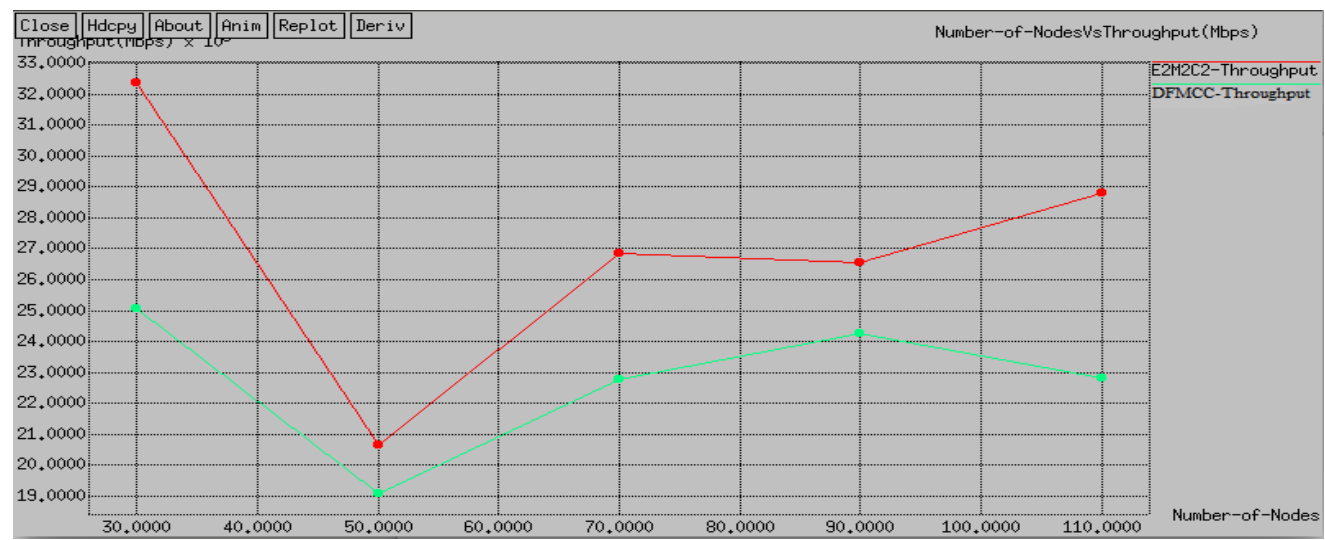

Figure 5. Throughput comparisons with impact of mobile users

Figure 6 shows the fairness index of proposed E2M2MC2 and existing DFMCC system. The plot clearly depicts the fairness index of proposed E2M2MC2 system is very high in terms of $44 \%$ high compared to existing DFMCC system.

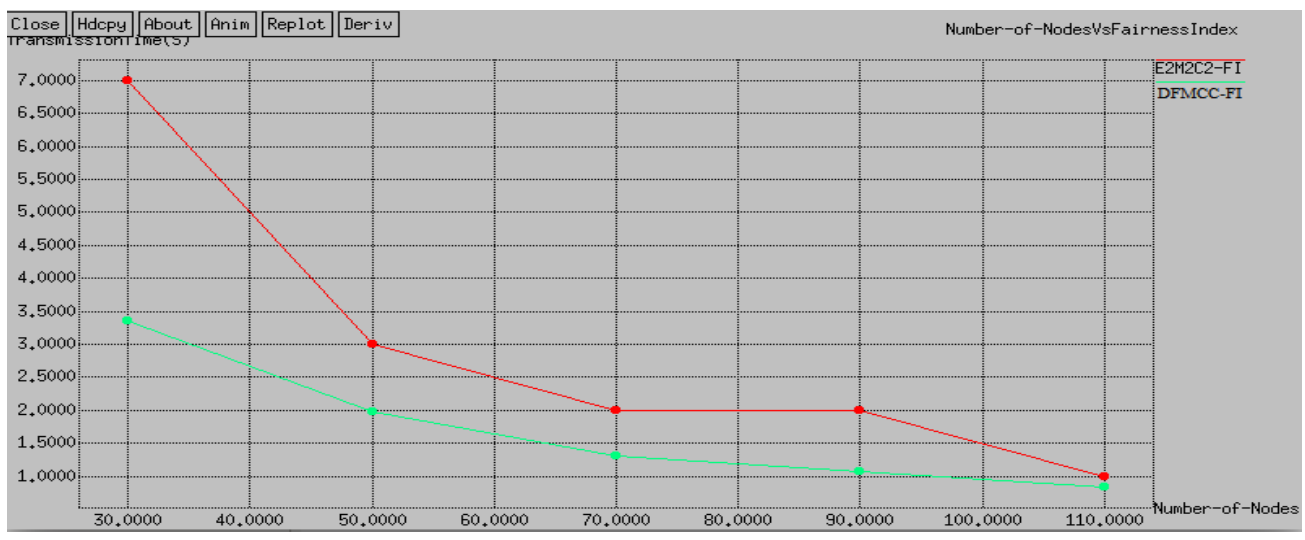

Figure 6. Fairness index comparisons with impact of mobile users 
Figure 7 shows the delay of proposed E2M2MC2 and existing DFMCC system. The plot clearly depicts the delay of proposed E2M2MC2 system is very low in terms of $20 \%$ high compared to existing DFMCC system.

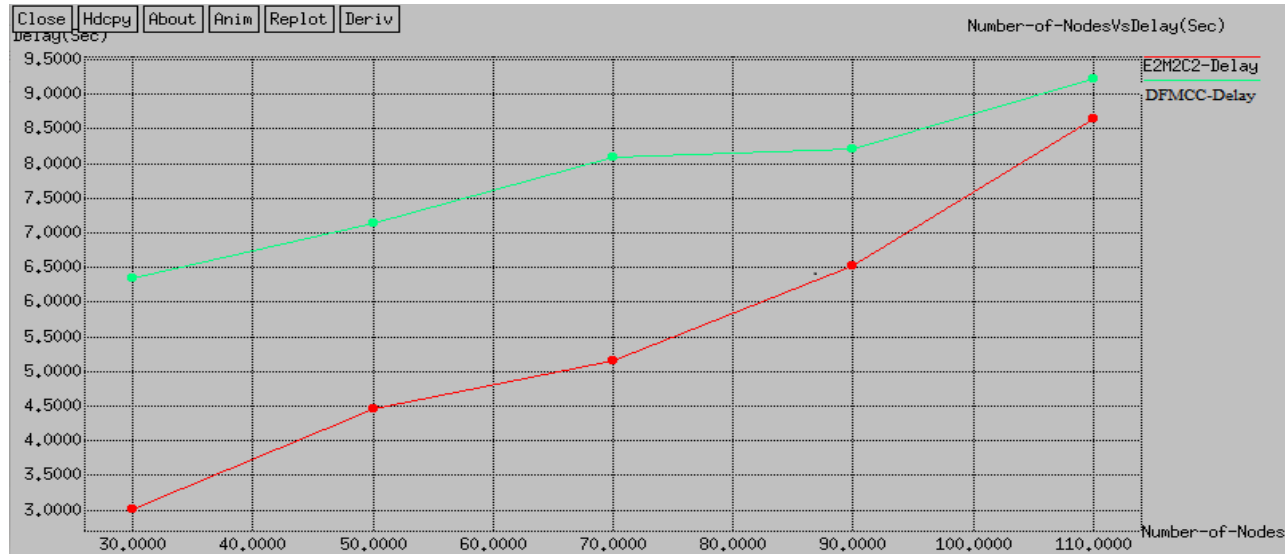

Figure 7. Delay comparisons with impact of mobile users

\section{CONCLUSION}

Mobile cloud computing makes use of the resources from the cloud to increase the computational capabilities of mobile devices. Mobile users can scale the available services to match their needs, customize apps and access cloud space from any location with an active internet connection. In this paper, we have proposed energy efficient mobility management in mobile cloud computing (E2M2MC2) system. It main objective is best cloud computation, which are achieved by the elective repeat multi-objective optimization (ERMO2) algorithm respectively. The simulation results prove the effectiveness of our E2M2MC2 system in terms of delay, packet loss rate, throughput, fairness-index and energy consumption.

\section{REFERENCES}

[1] J. Zhang, et al., "Towards Secure Data Distribution Systems in Mobile Cloud Computing," IEEE Transactions on Mobile Computing, vol/issue: 16(11), pp. 3222-3235, 2017.

[2] T. Taleb, et al., "On Multi-Access Edge Computing: A Survey of the Emerging 5G Network Edge Cloud Architecture and Orchestration," IEEE Communications Surveys \& Tutorials, vol/issue: 19(3), pp. 1657-1681, 2017.

[3] S. Namasudra and P. Roy, "Time saving protocol for data accessing in cloud computing," IET Communications, vol/issue: 11(10), pp. 1558-1565, 2017.

[4] H. S. Mansouri, et al., "Joint Optimal Pricing and Task Scheduling in Mobile Cloud Computing Systems," IEEE Transactions on Wireless Communications, vol/issue: 16(8), pp. 5218-5232, 2017.

[5] J. Zhang, et al., "Towards secure data distribution systems in mobile cloud computing," IEEE Transactions on Mobile Computing, vol/issue: 16(11), pp. 3222-3235, 2017.

[6] K. Kumar and Y. H. Lu, "Cloud Computing for Mobile Users: Can Offloading Computation Save Energy?" Computer, vol/issue: 43(4), pp. 51-56, 2010.

[7] P. Simoens, et al., "Remote Display Solutions for Mobile Cloud Computing," Computer, vol/issue: 44(8), pp. 46-53, 2011.

[8] V. Haghighi and N. S. Moayedian, "An Offloading Strategy in Mobile Cloud Computing Considering Energy and Delay Constraints," IEEE Access, vol. 6, pp. 11849-11861, 2018.

[9] W. Ren, et al., "Lightweight and compromise resilient storage outsourcing with distributed secure accessibility in mobile cloud computing," Tsinghua Science and Technology, vol/issue: 16(5), pp. 520-528, 2011.

[10] R. Barga, et al., "The Client and the Cloud: Democratizing Research Computing," IEEE Internet Computing, vol/issue: 15(1), pp. 72-75, 2011.

[11] S. Tursunova and Y. Kim, "Realistic IEEE 802.11e EDCA model for QoS-aware mobile cloud service provisioning," IEEE Transactions on Consumer Electronics, vol/issue: 58(1), pp. 60-68, 2012.

[12] J. Zheng, et al., "Component Importance Analysis of Mobile Cloud Computing System in the Presence of Common-Cause Failures," IEEE Access, 2018.

[13] W. C. Peng and M. S. Chen, "Query processing in a mobile computing environment: exploiting the features of asymmetry," IEEE Transactions on Knowledge and Data Engineering, vol/issue: 17(7), pp. 982-996, 2005. 
[14] Y. Satoh and K. Sakaue, "A secure and reliable next generation mobility," Synthesiology English edition, vol/issue: 2(2), pp. 107-120, 2009.

[15] W. Zhang, et al., "Energy-Optimal Mobile Cloud Computing under Stochastic Wireless Channel," IEEE Transactions on Wireless Communications, vol/issue: 12(9), pp. 4569-4581, 2013.

[16] W. Zhang, et al., "Toward a unified elastic computing platform for smartphones with cloud support," IEEE Network, vol/issue: 27(5), pp. 34-40, 2013.

[17] A. Tzanakaki, et al., "Virtualization of heterogeneous wireless-optical network and IT infrastructures in support of cloud and mobile cloud services," IEEE Communications Magazine, vol/issue: 51(8), pp. 155-161, 2013.

[18] N. V. Rodriguez and J. Crowcroft, "Energy Management Techniques in Modern Mobile Handsets," IEEE Communications Surveys \& Tutorials, vol/issue: 15(1), pp. 179-198, 2013.

[19] P. Tysowski and M. Hasan, "Hybrid Attribute- and Re-Encryption-Based Key Management for Secure and Scalable Mobile Applications in Clouds," IEEE Transactions on Cloud Computing, vol/issue: 1(20, pp. 172-186, 2013.

[20] E. Tilevich and Y. Kwon, "Cloud-Based Execution to Improve Mobile Application Energy Efficiency," Computer, vol/issue: 47(1), pp. 75-77, 2014.

[21] F. Sardis, et al., "On the Investigation of Cloud-Based Mobile Media Environments With Service-Populating and QoS-Aware Mechanisms," IEEE Transactions on Multimedia, vol/issue: 15(4), pp. 769-777, 2013.

[22] Q. Qi, et al., "Cloud service-aware location update in mobile cloud computing," IET Communications, vol/issue: 8(8), pp. 1417-1424, 2014.

[23] A. Gani, et al., "A review on interworking and mobility techniques for seamless connectivity in mobile cloud computing," Journal of Network and Computer Applications, vol. 43, pp. 84-102, 2014.

[24] S. Chen, et al., "Mobility-driven networks (MDN): from evolution to visions of mobility management," IEEE Network, vol/issue: 28(4), pp. 66-73, 2014.

[25] B. Hu, et al., "A Mobility-Oriented Scheme for Virtual Machine Migration in Cloud Data Center Network," IEEE Access, vol. 4, pp. 8327-8337, 2016.

[26] W. Junior, et al., "Supporting mobility-aware computational offloading in mobile cloud environment," Journal of Network and Computer Applications, vol. 94, pp. 93-108, 2017.

[27] S. Kim, et al., "Traffic management in the mobile edge cloud to improve the quality of experience of mobile video," Computer Communications, 2017.

[28] D. Seo, et al., "Cloud computing for ubiquitous computing on M2M and IoT environment mobile application," Cluster Computing, vol/issue: 19(2), pp. 1001-1013, 2016.

[29] A. Aissioui, et al., "Toward Elastic Distributed SDN/NFV Controller for 5G Mobile Cloud Management Systems," IEEE Access, vol. 3, pp. 2055-2064, 2015.

[30] Z. Che, "A multi-objective optimization algorithm for solving the supplier selection problem with assembly sequence planning and assembly line balancing," Computers \& Industrial Engineering, vol. 105, pp. 247-259, 2017. 